\title{
Enhancing and Monitoring Patient Outcomes Through Customized Learning
}

\author{
Majed Almotairi, Mohammed Abdulkareem Alyami, Yeong-Tae Song ${ }^{\text {}}$ \\ Dept. of Computer E Information Sciences, Towson University, 21252, USA
}

A R T I C L E IN F O

Article history:

Received: 21 December, 2018

Accepted: 31 January, 2019

Online: 13 February, 2019

Keywords:

Patient Education

Customized learning

Dublin Core Metadata

Learning Object

Learning Object Repository

\begin{abstract}
A B S T R A C T
Chronic diseases such as heart disease, cancer, diabetes, and asthma continue to increase in the general public within the modern era. With careful observation of the symptoms potential diseases may be detected early and managed properly. For that to happen, the awareness of the symptoms and proper knowledge about the diseases may be needed for each patient. To acquire such knowledge, patients may need to gather essential health information from a variety of sources such as the Internet, articles, or some type of e-learning systems. However, the amount of available information, often too much, which discourages patients to continue. In response to such scenarios, we propose an approach that delivers only the relevant information that is specific to each patient's condition. In our approach, we utilized observed symptoms and vital signs to identify potential diseases of a patient. As they use the system, their profile may be constructed to deliver patient-specific set of learning materials called a study plan. To monitor and promote their study, we developed a mobile application that allows patients to view their study plan(s) and to study the customized learning materials. Such customized learning allows patients to take control of their symptoms and potential diseases, which eventually helps them to improve their outcomes as a result.
\end{abstract}

\section{Introduction}

Chronic diseases such as heart diseases, cancer, diabetes, and asthma are continuously increasing [1]. As reported by the Centers for Disease Control and Prevention (CDC) [2], 60\% of adults in the US have one chronic disease, $40 \%$ have two or more chronic diseases, and almost $40 \%$ of adults were considered obese in 2015-2016. Annually, over 1.7 million people are diagnosed with cancer, and more than a third die from it. The US health care budget on cancer is incessantly increasing and anticipated to reach $\$ 174$ billion by 2020. Chronic diseases require continuous treatment, which decreases the quality of life and increase medical expenses. According to the CDC [1], about $90 \%$ of The US health care expenditure is spent on individuals with chronic diseases and mental health conditions. Hence, chronic diseases are considered highly costly compared to other health problems. However, these diseases may be prevented or improved by early detection and proper management. According to many of the randomized controlled trials, educational techniques may help decrease pain, increase coping skills, and decrease the primary care visits, which saves time and money $[3,4]$. Patients also need to learn about how and when to take their prescription drugs, and other information such as side effects. If patients do not follow the instruction on prescription, this may lead to undesirable results such as the status of disease getting worse, or even death, which eventually increases health care budget in the United States [5]. Therefore, it may help improve patients' outcome when care givers ensure that patients have read and understood the drugs' instructions.

When patients suffer from some symptoms such as chest pain, they would look for the information related to that. However, getting the proper knowledge about that can be quite difficult as patients may be overwhelmed by the vast amount of information that is available from various sources. They do not know where to start to educate themselves, as shown

*Yeong-Tae Song, Email:ysong@towson.edu 
in Fig. 1. CDC and WebMD, for instance, provide a vast amount of Internet accessible information regarding prescription drugs, diseases and their conditions, healthy living, public health statistics, and more that can be utilized by general public [2].

However, the efficient consumption of the available information by patients can be difficult due to the amounts and types of information. To overcome such issues, we propose an approach that filters out irrelevant information and provide only the necessary information for the patient, i.e., customized patient education. In our study, we deliver patient education by providing customized learning materials based on their prescription drugs, clinical conditions, and potential diseases. Therefore, only the relevant information that is specific to each patient is delivered. The learning materials are queried semantically and delivered to the patients from the cloud based learning object repository. To ensure effective learning, patients' levels of understanding in health information may be gained to control the difficulty level of learning materials. In particular, semantic queries can be performed based on a patient's clinical condition and learning needs. Then the obtained results from the queries are filtered and organized based on the patient's knowledge level and relevancy. Based on the query results, a study plan can be created and organized from the learning object repository, which satisfies the learners' needs. In addition, we employed an assessment technique to monitor the patient's level of understanding on the learning materials and to ensure that he/she has read and understood the content of the learning materials. The remainder of this paper is arranged as follows: Section 2 discusses the literature review. Section 3 gives an overview of the proposed Patient E- Learning System (PELS), discusses the role of the system components, and presents the implementation of the suggested system. Finally, we conclude our study in section 4 .

\section{Literature Review}

In the modern era, there are numerous contributions and researches about e-learning systems in various domains. For example, In [6], the authors offered a virtual medical school as an e-learning system that provides a problem-based e-learning environment that enables medical students and residents to access clinical cases by using the Hospital Information System (HIS). Ouf et al. [7] have used ontology and Semantic Web Rule Language (SWRL) to develop a smart e- learning ecosystem that delivers the associated research articles from different sources, such as IEEE library, Science Direct and Springer Link, to learners according to their needs (for instance preferred learning activities). In addition, other studies concentrated on ubiquitous learning [8-10], language learning, sharing of learning resources and mobile learning $[11,12]$. Mesquita and Peres [13] offered a Customized x-Learning Environment model that delivers customized learning materials by allowing learning to take place anytime
/ anywhere, depending on the student's needs and characteristics. However, the majority of the studies mentioned above rely on the learner profile generally in order to improve learner's achievements. Additionally, there is a lack of conducted studies on utilizing e-learning systems for patients and clinicians to use. Consequently, the nucleus of our research is to develop learning management system for clinicians and patients to use in order to improve patients health knowledge with an aim to improve patients outcomes. Despite the fact that there are many available resources (e.g. websites and mobile applications) that are reachable online for example PatientsLikeMe, MAYO Clinic, WebMD, iTriage, and others, patients are overwhelmed by the amount of learning materials. For example, PatientsLikeMe enables patients with the similar disease(s) to share information regarding their treatments, medical experiences, medication, outcomes, and more. However, room for medical errors exists while using this site since the patient is the one who generates the data. There are several reasons why these medical errors may take place, amongst them misconstruction due to limitation of using medical standards (e.g. patients may use of diverse terms to describe their problem), lack of using other factors that might be related to disease such as vital signs and demographic data, and concentration on diseases only [14]. In contrast, in our study, we utilized medical standards (e.g. RxNorm, SNOMED CT and ICD), vital signs and demographic data in order to provide learning materials to the patient according to his/her prescription drugs, diseases and symptoms and eventually avoid medical errors. In our study, an innovative learning approach is proposed by using customized learning. Based on this, a learning model is designed to achieve personalized learning experiences for patients. We can achieve this by retrieving the most proper learning objects and then providing them to proper patients. This, in turn, may encourage patients to educate themselves and reduce the time of seeking such learning materials from different resources.

\subsection{Related Standards}

This section describes the most common related standards that are available in the industry, and how they can be utilized in the PELS.

\subsubsection{Patient Profile}

In the modern era, there are various learner profile standards, including, but not limited to, FelderSilverman Model, Kolb's Experiential Learning Model and Myers-Briggs Type Indicator, each individual standard is suitable for a specific learning domain [15]. However, Luciana et al. [16], and Ioannis et al. [17] reported that the two widely held standards that are utilized to define the learner profile are: Public and Private Information (PAPI), introduced by Institute of Electrical and Electronics Engineers (IEEE) [18], and Learner Information Package (LIP), introduced 


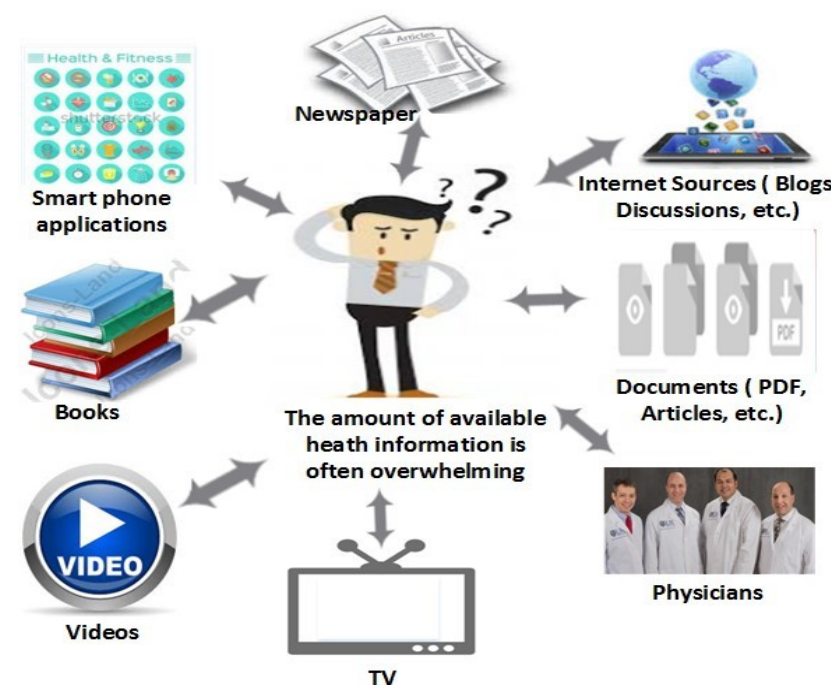

Figure 1: Patients are overwhelmed by the amount of available information

by Instructional Management System (IMS) [19]. The PAPI model contains six core classifications (personal information,security information, performance information, relation information, preferences information, and portfolio information) [18]. The IMS LIP model contains 11 core classifications (identification, affiliation, interest, accessibility, relationship, competency, activity, QCL [qualifications, certificates and license], goals, transcript, and security key) [19]. In addition, other extensions are available in each classification of these two standards that enable dealing with more possible characteristics $[18,19]$. Evangelou et al. [20] expresses that the PAPI model is generic and was developed to be utilized by variety types of systems or applications, but it does not afford information about dynamic characteristics of the learner profile. However, LIP is useful in the case that the system needs to be interoperable with other systems [20]. As mentioned above, the PAPI and LIP models comprise different categories to describe the learner profile, but none of these models entirely meet the PELS requirements, such as allowing clinicians to input their patients' symptoms and vital signs regularly. For that to happen, we developed a patient profile that involves specific characteristics to describe both dynamic and static information for each patient, which are utilized to gather patients' information in order to deliver customized learning materials to each individual.

\subsubsection{Learning Object}

A variety of learning object (LO) metadata standards are available in industry that are utilized to describe the LOs, which in turn, enables the ease of discovery and retrieval of LOs. An example of these standards, Learning Object Discovery and Exchange (LODE), was introduced by IMS Global (www.imsglobal.org). Another example of the standards, is called the Learning Object Metadata (LOM) developed by IEEE, which offers a technique to de- scribe the profile of the LOs for use in e-learning systems [21]. Furthermore, one of the most common standards that is available is called the Dublin Core (DC), which also can be utilized to represent the LO profiles [22]. For generating and packaging the LOs, a wellknown standard is called Common Cartridge (CC), which offers a standardized technique to create the LO content [23]. The CC also provides a standardized way to make interoperable e-learning systems and learning materials [23]. The CC utilizes the core components of the DC to describe the LO [22]. In our research, we utilized the CC standards that best fit our purpose. Most of the well-known e-learning systems use the CC to create the LOs as it offers standard methods to create LOs.

\subsubsection{Medical Standards}

Alyami et al. [24] summarizes the medical standards that are used at the present time. The ones that are related to our study are:

- Systemized Nomenclature of Medicine Clinical Terms (SNOMED CT): a comprehensive standardized clinical terminology [24].

- International Classification of Diseases (ICD9/ICD-10): used for epidemiology, health management and clinical purposes [24].

- RxNorm: used to provide normalized names for clinical drugs [24].

These three standards are used in the PELS for the clinicians use when they input data in the patients' profile (e.g. symptoms and prescription drugs).

\subsubsection{Function Modeling Notations:}

The US Air Force Program for Integrated Computer Aided Manufacturing (ICAM) presented various modeling notations that they have developed to provide better analyses and communication techniques in order to increase the manufacturing efficiency [25]. The main modeling notation that ICAM presented is called IDEF0 (ICAM Definition), which is based on Structured Analysis and Design Technique TM (SADTTM) [25]. IDEF0 is used to create a functional model that represents the functions, activities and processes that are involved in a system [25].

\subsection{Limitations of Existing E-learning Systems}

We examined the contemporary e-learning systems that are available for the public in industry, and the results show that there are more than 400 e-learning systems, either commercial, free or open source systems. In order to obtain functions that can be utilized for clinical education appropriately, we utilized the main functions from the free and open source well-known learning management systems, including Moodle, ATutor, Dokeos, ILIAS and Sakai. In addition, we took into 


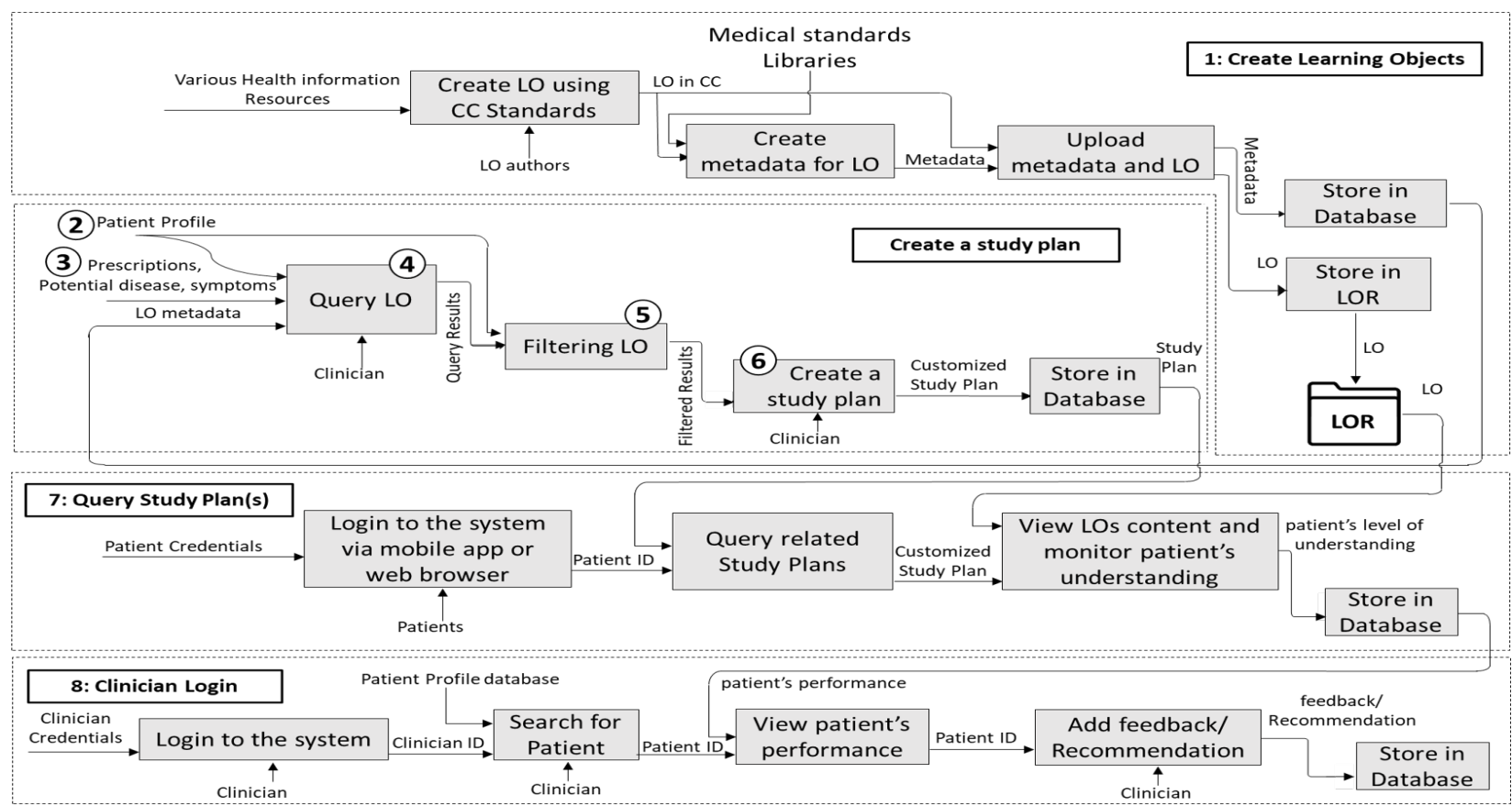

Figure 2: Main Functions of the Patient E-learning System (Numbers: 1, 2, etc. in this figure correspond to section 3)

account the other organizations that offer information for patients, such as CDC, WebMD and MAYO Clinic. The results indicate that there are many limitations of these systems, including:

- In the most of the e-learning systems, the instructor chooses particular content to be accessible by learners, which make the system a teachercentered instruction.

- Limited options are available for customized learning or filtering for learning materials that can be utilized to satisfy learners' needs and goals. However, patients are required to use the search function within these e-learning systems to query the desired information.

- Enrollment is required in most cases by each individual learner in a specific course to be able to contribute and view the course materials where the instructor is the person who evaluates the students' performance.

- Limited development of learner profiles that may allow for saving and analyzing the current learner's condition in order to offer learning materials based on their needs.

- MAYO and WebMD organizations have developed web applications that deliver clinical information to the users according to their inputs (e.g. prescription drugs and symptoms). However, they do not provide any assessment techniques to ensure that users have read and understood the content. In addition, they do not enable patients to save their information so they can monitor the changes of the patient outcomes.
To cope with these boundaries and limitations, we proposed new functions and improved some of the functions of existing applications to be used in the PELS as follows:

- To generate the learning object, we followed the CC standard for the creation of the learning objects, and we used the DC metadata to represent the learning object in order to facilitate the finding and retrieval of learning materials that are associated with the patients' education.

- As none of the current learner profiles that exist in the current e-learning systems are appropriate for the patient education, we offered a patient profile with specified characteristics that can deal with static and dynamic information, such as patient's vital signs and symptoms. We utilized patient profiles to enable the following:

- To allow clinicians to save and update patients' information (e.g. symptoms and potential disease[s]).

- Dynamic retrieval of customized learning materials that meet patients' needs according to the available information in patients' profiles. Therefore, our proposed system is not similar to those that ask users to type keywords in the search textbox to retrieve their desired learning materials.

- Assessment techniques are conducted to ensure that patients have read and understand the learning materials. 


\section{Create a new exam}

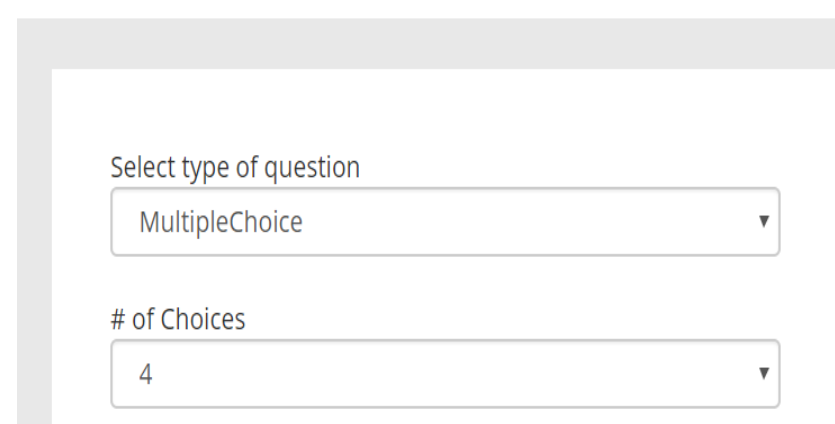

Continue.

Figure 3: Exam creator tool for LO authors

- For the ease of use, we developed a mobile application that allows patients to view their learning materials straightforwardly.

- Although the PELS is an independent system, well-known standards (e.g. medical and metadata standards) were utilized to record such data in our proposed system. Therefore, the data can be integrated with other systems if they utilize the same standards.

\section{The Proposed Patient Learning System (PELS)}

Patients and clinicians are the main users in our proposed PELS. For that reason, the limitations of the systems that provide information for patients and the current e-learning systems are taken into account. In addition to the functionality of our proposed system that was presented in [1], we also proposed more enhancements by adding more functions that are used to deliver learning materials to patients based on their need and capture their performance in order to insure that patients have learned the desired knowledge. To represent the functionality of the PELS, the IDEF0 notation [25] has been used to show the main components of the proposed system along with the main input(s) and output(s) of each component as shown in Figure 2. The following sections illustrate in detail how each component is used in the PELS.

\subsection{Creating Learning Objects}

We utilized the CC standard [23] in the process of creating the LOs which make the PELS organize and retrieve the LOs efficiently. In addition, the CC standard utilizes the DC standard for the metadata representation. The processes of creating LO are shown in the Figure 2 section 1.First, the LO authors create LO using

\section{Confirmation!}

The exam has been Created Successfully!

Please copy the link below and paste it at the end of the learning object content.

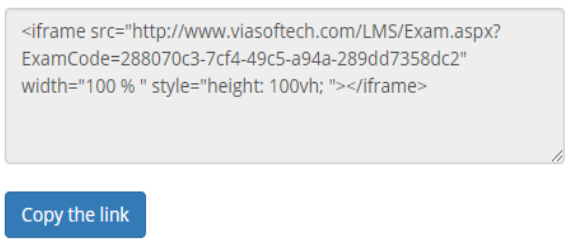

Figure 4: Example of the generated embed code

any content authoring tool that create LO in CC standards. While creating the LO, most of the content authoring tools allow authors to create exams and quizzes for each LO to ensure that learners have read and understood the content of the LO.

However, by using these authoring tools, it is difficult for the PELS to capture and save patient's answers, as there is no option to integrate LOs with the PELS (or third party systems in general) that allow developers to capture learners' inputs and store them in a database. Therefore, we developed an exam creator tool that allows LO authors to create an exam that can be managed by each clinician to capture their patients' performance. However, patients may not be able to answer questions that require typing, therefore, we only E- focused on multiple-choice and True/False questions that are easy for patients to answer and for the clinician to receive the final score without reading through the responses.

To create an exam, the $\mathrm{LO}$ authors create a title for the exam as first step, and then they choose the type of question(s) which can be either multiple-choice or True/False. If the author chooses a multiple-choice question, another menu appears that allows them to specify the number of choices as shown in Figure 3. The LO author can repeat this step several times to add the desired number of questions they want. When they finish adding questions, the exam creator tool generates an embed code that allow LO authors to embed the exam with the LO as shown in Figure 4. Once LO authors complete the process of creation the LO, they need to add metadata based on the DC standard to represent the LO. DC consists of fifteen core elements that can be used to define the LO profile more precisely [22]. In this research, we used the DC metadata elements to describe clinical LOs efficiently, as shown in Table 1. The LO authors are required to fill most of these elements in order to gain metadata records that can be queried efficiently. An example of the learning objects' metadata that are stored in the database is shown in Figure 5. The World Health Organization (WHO), that provides International Classification of Diseases (ICD9/ICD-10), grouped the potential diseases based on the body systems and other factors. In our study, we 
Table 1: Metadata schema for the LO

\begin{tabular}{|l|l|}
\hline Element & Description \\
\hline Title & Learning object's title \\
\hline Creator & Learning object's author \\
\hline Subject & Keywords that outlines the LO \\
\hline Description & Detailed description about the LO \\
\hline Relation & $\begin{array}{l}\text { The related medical standard code(s) } \\
\text { that is related to the topic of the LO, } \\
\text { which also can be used to indicate } \\
\text { whether the LO is specific or generic }\end{array}$ \\
\hline Date & The creation date of the learning object \\
\hline Type & $\begin{array}{l}\text { The category of the LO ( e.g. symptoms, } \\
\text { causes, diagnosis and drugs) }\end{array}$ \\
\hline Identifier & The ID of the learning material \\
\hline Language & $\begin{array}{l}\text { The spoken/written language used } \\
\text { in the content of the LO (e.g. English } \\
\text { and Spanish) }\end{array}$ \\
\hline Rights & Access rights and copyright of the LO \\
\hline Source & The source of the LO \\
\hline
\end{tabular}

utilized these classifications to determine whether the LO is generic (e.g. body system) or specific (e.g. specific disease).

The PELS utilizes the Learning Object Repository (LOR), which is a directory that is used to organize and store all learning materials. Accordingly, once the LO authors complete filling the metadata of a LO and click "upload metadata and LO", the system creates a separate sub-directory inside the LOR for each new LO with a unique label and adds this unique label to the LO's metadata to be efficiently and effectively queried.

The content of the LOs can be collected from various recognized health information resources. However, the creation of the LO can be collected manually using any e-learning content authoring tool that generates $\mathrm{LO}(\mathrm{s})$ in CC standard. In addition, other organizational websites (e.g. CDC [2]) provide Syndication API, that collect an enormous number of learning materials from their repository. All learning materials are stored and organized in the LOR to allow the PELS to use them and retrieve appropriate ones for the patient efficiently. During the exploratory analysis, we collected content for variety types of diseases, symptoms and drugs from different well-known health information resources such as CDC [2] and created LOs based on the CC standard.

\subsection{Patient Profile}

The Patient profile is developed to store patients' information regularly. As patients' information may include clinical information such as prescriptions, symptoms and vital signs, clinicians are involved in the process of adding the clinical information to the patient profile. In this study, we categorized the patient profile into two groups: dynamic (clinical information) and static (demographic information) information for each patient. The PELS utilizes patient profiles to gather information about each patient in order to deliver customized learning materials to each individual patient. The components of the patient profile can be listed as

\begin{tabular}{|l|l|l|l|}
\hline Id & Title & Description & Lang... \\
\hline 1 & Genital Warts I S... & Genital warts ... & EN \\
\hline 2 & How to prevent ... & Pertussis (wh... & EN \\
\hline 3 & Vitiligo: Get the F... & Vitiligo is a co... & EN \\
\hline 4 & Strep Throat: Sy... & Strep throat is... & EN \\
\hline 5 & Key Facts About ... & Influenza (als... & EN \\
\hline 6 & Pink Eye Facts:... & "Pink eye" is ... & EN \\
\hline 7 & The Best and W... & Picking the ri... & EN \\
\hline 8 & Acute Maxillary .... & Acute sinusiti... & EN \\
\hline 9 & Scabies: Images.... & Scabies is a v... & EN \\
\hline 10 & What Causes Kid... & Kidney stones... & EN \\
\hline
\end{tabular}

Figure 5: Portion of the learning objects metadata

follows (see Figure 6):

- Patient demographic information: consists of full name, date of birth, gender, address, username, password, email address and phone number.

- Patient level of knowledge and preference: Level of knowledge is used to determine the learning materials' difficulty level that patient can understand. Preference contains content types such as text, video, etc., preferred language and input / output device.

- Patient vital signs: updated frequently, which includes respiratory rate, pulse, temperature, blood oxygen saturation and blood pressure.

- Patient symptoms: includes symptoms that patients observe, which may be used to determine the potential disease(s). Potential diseases: includes disease(s) that patients may have based on their symptoms.

- Prescriptions: includes prescription drugs that patients obtained.

- Study Plan: a collection of customized LOs for each individual patient to study.

On the other hand, clinicians' profile consists of the following:

- Demographic information: consists of full name, date of birth, gender, address, username, password, email address and phone number.

- Patient list: a list of patients that the clinician deals with. In order for the clinician to add a patient in their list, the patient's approval is required.

By using PELS, a patient signs up as a new patient and starts adding his/her main information and preferences (static information). However, patients may not be able to deal with clinical information. Therefore, 


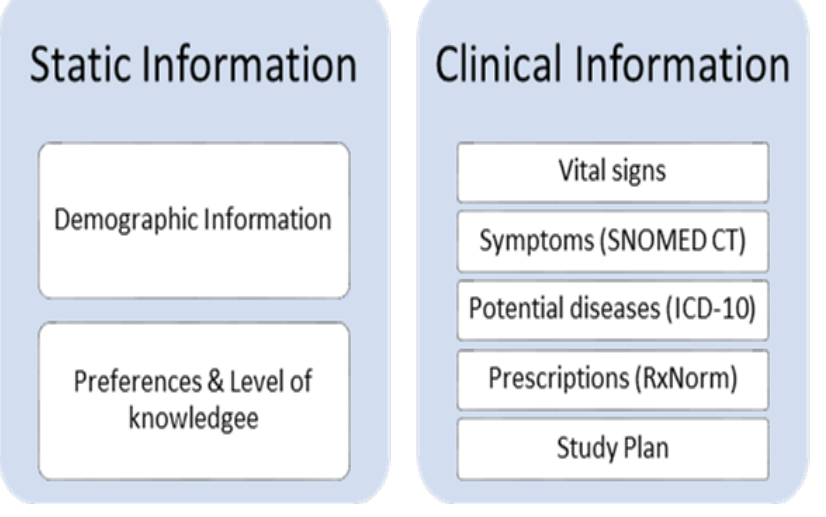

Figure 6: Components of the Patient Profile

the PELS limits adding clinical information only to clinicians. Consequently, a clinician needs to search for a patient first using search criteria, and then sends a request to the patient to add him/her to their list. The patient then will have the option to approve/reject the clinician's request. Once the patient approves the clinician's request, the clinician then can add clinical information to the patient profile.

\subsection{Potential Diseases and Prescriptions}

With the aim of creating study plans, the PELS needs to determine the potential diseases that patients may have, observed symptoms that they are suffering from, or prescription drugs that clinician prescribed. To collect such data, potential diseases can be entered in two methods: directly and indirectly. From the clinician directly, if the clinician already know the (potential) disease that patient suffers from. Indirectly, which can be obtained by using the Health Decision Support System (HDSS) that we have developed [24] which relies on medical standards such as SNOMED CT and ICD-9/ICD-10. HDSS assists patients and clinicians in identifying such potential disease(s) based on symptom(s) they provided. For instance, if a patient utilizes HDSS to determine his/her potential disease and enters his/her symptoms through the system, PELS can store this information on the patient profile, as shown in Figure 7. On the other hand, the prescription drug(s) need to be determined in the patient profile in order to educate patients about how to use specific drug, side effects when using this drug, and more. The PELS utilizes the RxNorm standard that allows clinicians to utilize standard terms for clinical drugs when adding them to the patient profile.

\subsection{Query Module}

As patients may not know how to deal with clinical terms or what is the right learning materials they should start with, clinicians are involved in this process to make sure that patients receive learning materials based on their needs. To query the metadata, Microsoft offers the semantic search that built upon the full-text

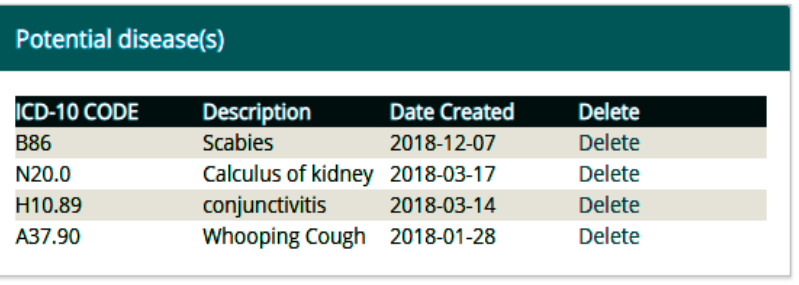

symptom(s)

\begin{tabular}{|llll|}
\hline SNOMED CT CODE & Description & Date Created & Delete \\
\hline 128870005 & $\begin{array}{l}\text { Crusted scabies } \\
\text { (disorder) }\end{array}$ & $2018-12-07$ & Delete \\
\hline 95570007 & Kidney Stones & $2018-03-17$ & Delete
\end{tabular}

Figure 7: List of potential diseases and symptoms in the patient profile

search feature in the SQL server [26]. It extracts and statistically indexes the relevant key phrases. These key phrases are used to find and index the similar or related results [26].

The SQL server provides mechanisms that are utilized to implement the full-text search, for instance [26]:

- Stop list: deals with list of words commonly taking place in strings that do not help the search which should to ignored when performing a query e.g. "in" or "an".

- Stemmer: deals with the inflectional forms of a word, for instance, in the word "play" the fulltext searches for the stems of the word, such as played, playing and player.

- Thesaurus: deals with the synonyms of a word, for example bike and bicycle.

- Word breaker: deals with the word boundaries as in the words "multi-millions" and "multimillions".

- Replacements: deals with the most frequently misspelled words such as Calendar/ Calender.

The clinician manages this process in order to ensure that patient receives the desired learning materials. In this way, amongst variety kinds of LOs that are stored in the LOR, and based on the available information in the patient profile, the PELS analyzes this information through the query module in order to deliver the proper learning materials to the patient. To do so, this module systematically queries the most associated learning materials that the patient needs based on his/her conditions. The query results (retrieved LOs) go through to the filtering module.

\subsection{Filtering Module}

The filtering module gets called after the query module is executed in order to retrieve customized learning materials based on the patient's profile. It filters the learning materials according to the patient's 


\begin{tabular}{|c|c|}
\hline Learning Object Title & $\begin{array}{l}\text { View } \\
\text { Contents }\end{array}$ \\
\hline $\begin{array}{l}\text { How to prevent Pertussis (Whooping Cough) ( Not Completed!) } \\
\text { This Learning material is recommended to you because it is associated with the } \\
\text { potential disease Whooping Cough that is listed in your profile }\end{array}$ & $\begin{array}{l}\text { View } \\
\text { Contents }\end{array}$ \\
\hline $\begin{array}{l}\text { Pink Eye Facts: Identify Symptoms and Treat Pink Eye (Conjunctivitis) ( New!) } \\
\text { This Learning material is recommended to you because it is associated with the } \\
\text { potential disease Conjunctivitis that is listed in your profile }\end{array}$ & $\begin{array}{l}\text { View } \\
\text { Contents }\end{array}$ \\
\hline $\begin{array}{l}\text { Key Facts About Influenza (Flu) | Seasonal Influenza (Flu) (Completed!) } \\
\text { This Learning material is recommended to you because it is associated with the } \\
\text { potential disease Influenza (disorder) that is listed in your profile }\end{array}$ & $\begin{array}{l}\text { View } \\
\text { Contents }\end{array}$ \\
\hline
\end{tabular}

Figure 8: Customized study plan based on patient profile

preferences and/or the level of knowledge. It also eliminates learning materials that the patient has already completed in the past. In this manner, the module retrieves only the related learning materials for an individual patient, i.e., customized learning contents, to the patient.Figure 5 and Figure 8 show the differences before and after filtering.

\subsection{Creating Study Plans}

The study plan is a collection of learning materials that patients can study to educate themselves about a certain topic [27]. In the PELS, the clinician is involved in this process to verify the process of selecting learning materials. The created study plan module automatically generates a collection of learning materials according to the results received from the query and filtering modules in order to provide it to the patient. Then clinicians can verify the choices of learning materials as they have the authorization of adding/deleting learning materials from the study plan. When the clinician confirms the creation of the study plan, the PELS generates a list of recommended learning materials as an XML that contains information about the recommended learning materials, and it points out the reason of recommending each learning material.Figure 8 shows how the patients view the content of the study plan in PELS. The PELS allows multiple creations of study plans. Therefore, each time that clinicians update patients' conditions (e.g. add new potential disease), this module allows clinicians to generate a new study plan that contains a new list of learning materials in order to provide efficient learning materials to each individual patient according to their up-to-date conditions.

\subsection{Query Study Plan}

This module is about how the PELS queries the study plan(s) and delivers them to the right patient. Patients may not like the way that they need to use the personal computer/laptop to be able to sign in to the PELS and study. Therefore, we make the use of the

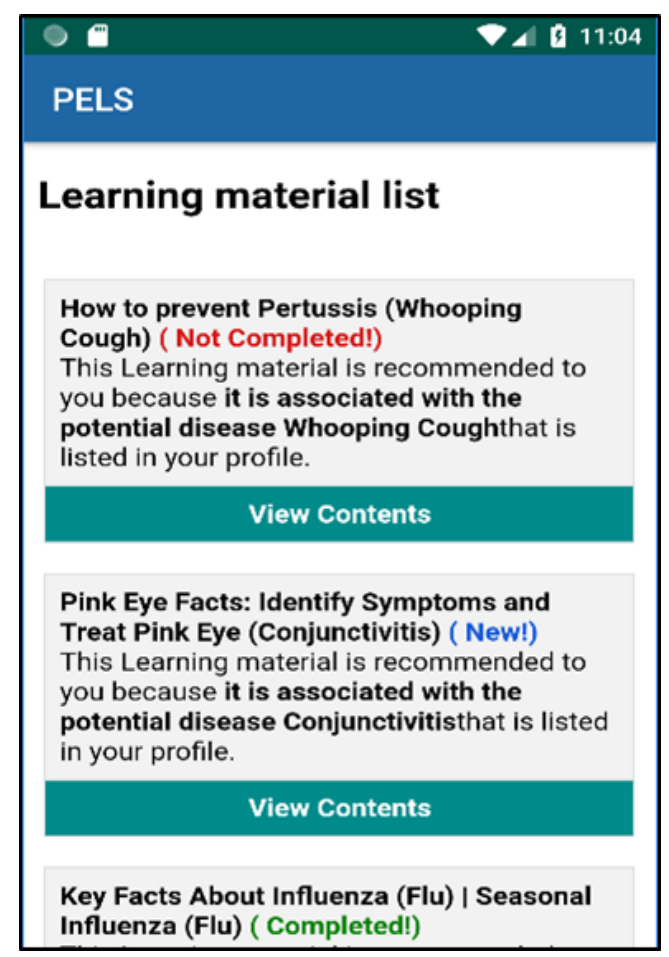

Figure 9: View study plan content through the mobile application

PELS even easier by developing a mobile application that allows them to view and navigate through their study plan(s) and display the content of each study plan. Figure 9 shows how patients view study plan(s) when they use the mobile application. In order for the PELS to query the patient's study plan(s), the patient first needs to log in using their credentials. Once the patient logs in successfully, the PELS queries the list of study plan(s) associated with this particular patient. Each study plan includes one or more learning material(s) that are approved by a clinician as shown in Figure 8. In order to monitor how patients consume the learning materials, the PELS categorizes three different statuses that appear next to the title of the learning object as shown in Figure 8. The time when each of these three statuses that appear can be illustrated as follows:

- New: appears when the patient still has not viewed the learning material yet.

- Not Completed: each learning material contains a quiz that needs to be completed by the patient to ensure that he/she has read and understand the content of the learning material. This status appears when the patient viewed the learning material but still has not attempt to complete the quiz.

- Completed: appears when the patient spent some time to reading the learning material and has completed answering the quiz.

If the status of the learning material remains new or not completed for a certain period of time (e.g. a week), 
the system sends a reminder notification to the patient saying that the learning material needs to be completed. However, at the end of each learning material

\section{Patient Name: \\ Study Plan Title: Flu, and other symptom \\ Total Learning materials in the Study Plan: 3 Learning material(s)}

\section{Current Progress for each Learning material}

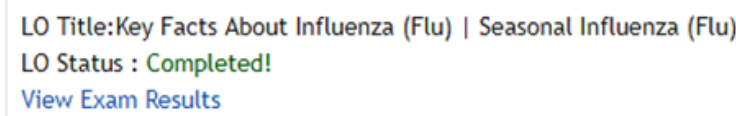

Figure 10: Patient's performance from the clinician's view

content, there is a quiz, which has been included to encourage patients to read the learning materials and make sure that they understand its content. Therefore, the PELS allows patients to take the quiz multiple times if needed. Accordingly, when the patient chooses the wrong answer, the PELS shows a hint to tell the patient that he/she needs to change their response and find the correct answer. On the other hand, when the patient views the content of the learning material, the PELS contains quantitative measures that capture the patient's performance of metrics, for instance:

- Total time that the patient spent on reading the learning material.

- Total number of attempts that the patient made to complete the quiz.

- Total points scored on each attempt.

All the captured data are stored in the patient profile so the patient can see how he/she performed on each task. In addition, it can reachable by the clinician so they can see how their patient performed in completing their tasks (see next section).

\subsection{Clinician Login}

Clinicians need to have their own access to the PELS so they can perform particular tasks, such as creating study plans and monitoring their patients' performance. Therefore, the PELS provides certain views with their functionality that perform when the user signs in as a clinician. Accordingly, clinicians start signing in to the PELS using their credentials if they have already created their own account. If they want to deal with a new patient, they need to search for the patient using the search criteria and send a request to the patient to add him/her to their list. If the patient approves the request, the patient will be added to the clinician list. Accordingly, the clinician can select any patient from their list in order to perform any task

\begin{tabular}{|l|l|}
\hline $\begin{array}{l}\text { Time spent on reading the Learning Material } \\
\text { Total attempts to complete the quiz }\end{array}$ & 2 attempt(s) \\
\hline Attempt \# 1 & $11 / 17 / 20189: 19: 27$ PM \\
\hline Submission Time & $2 /$ out of 8 \\
\hline Total points scored & \\
\hline Attempt \# 2 & $11 / 17 / 20189: 34: 11 \mathrm{PM}$ \\
\hline Submission Time & $8 /$ out of 8 \\
\hline Total points scored
\end{tabular}

Figure 11: Patient's quiz result

such as adding a new potential disease(s) and recommending study plan(s). In addition, clinicians can view patients' performance and see how they performed in a specific recommended study plan as shown in Figure 10. The PELS shows the status of each learning material whether or not the patient has read the learning material, has read the learning material but still has not completed the quiz or if the patient has completed reading the learning material and has answered the quiz. When the status is completed, the PELS shows an option to view the results, which includes the metrics that are mentioned in the previous section, shown in Figure 11. However, the PELS has an optional feature that allows clinicians to add feedback/recommendation for their patient when needed.

\section{Conclusion}

In this paper, we provide customized e-learning for patients with the aim of increasing their level of health knowledge and eventually improving their outcomes. For this to happen, we analyzed patient health data, such as observed symptoms and vital signs, to identify potential disease(s) for the given health data. Such factors are utilized to build patient profiles (such as patient preferences) that are used to provide only the relevant learning materials. Our proposed system, PELS, showed how to gather and organize heterogeneous learning materials using CC and DC metadata. To facilitate the retrieval of relevant LOs, the DC tags are utilized to retrieve such learning materials. In addition, we use an assessment technique to ensure that patients have read and understood the content of the learning materials. Moreover, we enhanced the usability of the PELS by developing a mobile application to facilitate the ease of use. Through this approach, we provide customized education that may help to improve patient outcomes . For future work, we plan to utilize an ontology-based patient profile analysis, which may increase the level of accuracy and relevancy of the search. 


\section{References}

[1] This paper is an extension of work originally presented in 2018 IEEE/ACIS 19th International Conference on Software Engineering, Artificial Intelligence, Networking and Parallel Distributed Computing (SNPD) Busan, 2018. http://dx.doi.org/10.1109/SNPD.2018.8441054.

[2] CDC, "Centers for disease control and prevention," https://www.cdc.gov/, 2006, accessed: 2017-01-21

[3] K. Jordan, N. Arden, M. Doherty, B. Bannwarth, J. Bijlsma, P. Dieppe, K. Gunther, H. Hauselmann, G. Herrero-Beaumont, P. Kaklamanis et al., "Eular recommendations 2003: an evidence based approach to the management of knee osteoarthritis: Report of a task force of the standing committee for international clinical studies including therapeutic trials (escisit)," Annals of the rheumatic diseases, vol. 62, no. 12, pp. 1145 1155, 2003. doi:10.1136/ard.2003.011742.

[4] E. Superio-Cabuslay, M. M. Ward, K. R. Lorig et al., "Patient education interventions in osteoarthritis and rheumatoid arthritis: a meta-analytic comparison with nonsteroidal antiinflammatory drug treatment," Arthri- tis Care and Research, vol. 9, no. 4, pp. 292301, 1996. DOI: 10.1002/15290131(199608)9:43.0.CO;2-4.

[5] M. L. McCarthy, R. Ding, N. K. Roderer, D. M. Steinwachs, M. J. Ortmann, J. C. Pham, E. S. Bessman, G. D. Kelen, W. Atha and R. Retezar, "Does providing prescription information or services improve medication adherence among patients discharged from the emergency department? A randomized controlled trial," Annals of emergency medicine, vol. 62, no. 3, pp. 212-223, 2013. DOI: 10.1016/j.annemergmed.2013.02.002.

[6] F.-M. Shyu, Y.-F. Liang, W.-T. A. Hsu, J.-J. Luh, and H.-S. Chen, "A problem-based e-learning prototype system for clinical medical education," in Medinfo, 2004, pp. 983987. DOI: 10.3233/978-1-60750-949-3-983.

[7] S. Ouf, M. A. Ellatif, S. E. Salama, and Y. Helmy, "A proposed paradigm for smart learning environment based on semantic web," Computers in Human Behavior, vol. 72, pp. 796818, 2017. https://doi.org/10.1016/j.chb.2016.08.030

[8] S. Yu, X. Yang, G. Cheng, and M. Wang, "From learning object to learning cell: A resource organization model for ubiquitous learning," Journal of Educational Technology \& Society, vol. 18, no. 2, p. 206, 2015.

[9] G.-J. Hwang, C. Hui-Chun, S. Ju-Ling, S.-H. Huang, and T. Chin-Chung, "A decision-tree-oriented guidance mechanism for conducting nature science observation activities in a context-aware ubiquitous learning environment," Journal of Educational Technology \& Society, vol. 13, no. 2, p. 53, 2010.

[10] Y.-M. Huang and P.-S. Chiu, The effectiveness of the meaningful learning-based evaluation for different achieving students in a ubiquitous learning context, Computers \& Education, vol. 87, pp. 243253, 2015. https://doi.org/10.1016/j.compedu.2015.06.009.

[11] Y.-J. Lan and Y.-T. Lin, "Mobile seamless technology enhanced csl oral communication." Journal of Educational Technology \& Society, vol. 19, no. 3, 2016.

[12] J. Abramson, M. Dawson, and J. Stevens, "An examination of the prior use of e-learning within an extended technology acceptance model and the factors that influence the behavioral intention of users to use m- learning," SAGE Open, vol. 5, no. 4, p. 2158244015621114, 2015. https://doi.org/10.1177\%2F2158244015621114
[13] F. Moreira, A. Mesquita, and P. Peres, "Customized x-learning environ- ment: Social networks \& knowledge-sharing tools," Procedia Computer Science, vol. 121, pp. 178185, 2017. https://doi.org/10.1016/j.procs.2017.11.025

[14] J. H. Frost and M. P. Massagli, "Social uses of personal health infor- mation within patientslikeme, an online patient community: what can happen when patients have access to one anothers data," Journal of medical Internet research, vol. 10 no. 3, 2008. https://dx.doi.org/10.2196\%2Fjmir.1053

[15] R. M. Felder and R. Brent, "Understanding student differences," Journal of engineering education, vol. 94, no. 1, pp. 5772, 2005. https://doi.org/10.1002/j.2168-9830.2005.tb00829.x

[16] L. A. Zaina, J. F. Rodrigues Jr, and G. Bressan, "An approach to design the student interaction based on the recommendation of e-learning objects," in Proceedings of the 28th ACM International Conference on Design of Communication. ACM, 2010, pp. 223228. https://doi.org/10.1145/1878450.1878488

[17] I. Panagiotopoulos, A. Kalou, C. Pierrakeas, and A. Kameas, "An ontology-based model for student representation in intelligent tutoring systems for distance learning," in IFIP International Conference on Artificial Intelligence Applications and Innovations. Springer, 2012, pp. 296305. https://doi.org/10.1007/978-3-642-33409-2_31

[18] IEEE, "IEEE standard for learning technology - public and private information (papi) for learners," http://metadatastandards.org/, 2002, accessed: 2017-09-04.

[19] IMS Global Learning Consortium Inc, "Ims learner information packaging infomation model specification," http://www.imsglobal.org/profiles/lipinfo01.html, 2001, accessed: 2017-09-07.

[20] C. E. Evangelou, M. Tzagarakis, N. Karousos, G. Gkotsis, and D. Nou- sia, "Augmenting collaboration with personalization services," Inter- national Journal of Web-Based Learning and Teaching Technologies (IJWLTT), vol. 2, no. 3, pp. 7789, 2007. DOI: $10.4018 /$ jwltt.2007070105

[21] IEEE, "IEEE standard for learning object metadata," https://standards.ieee.org/findstds/standard/1484.12.12002.html, accessed: 2017-10-09.

[22] DCMI, "innovation in metadata design, implementation \& best prac- tices," http://www.dublincore.org/documents/dces, accessed: 2017-10-02.

[23] IMS Global, "Ims common cartridge specification," http://www.imsglobal.org/activity/common-cartridge, 2008, accessed: 2017-11-12.

[24] M. A. Alyami, M. Almotairi, A. R. Yataco, and Y.-T. Song, "Health decision support system based on patient provided data for both patients and physicians use," Presented in ACM IMCOM 2018,12th International Conference on Ubiquitous Information Management Information Man- agement and Communication. https://doi.org/10.1145/3164541.3164632

[25] F. PUBS, "Announcing the standard for integration definition for func- tion modeling (idef0)," Draft Federal Information Processing Standards Publication, vol. 183, 1993.

[26] Microsoft, "Semantic search (sql server)," https://docs.microsoft.com/en- us/sql/relationaldatabases/search/semantic-search-sql-server, accessed: 2017-06-12.

[27] Y.-T. Song, K.-e. Park, and Y. Yoon, "Ontology based learnercentered smart e-learning system," in Proceedings of VI International GUIDE Conference, Athens, 2013. 